\title{
Empowering Active Learning to Jointly Optimize System and User Demands
}

\author{
Ji-Ung Lee \\ Christian M. Meyer \\ Iryna Gurevych \\ \{lastname\}eukp. informatik.tu-darmstadt.de \\ Ubiquitous Knowledge Processing (UKP) Lab \\ Computer Science Department \\ Technische Universität Darmstadt, Germany \\ https://www.ukp.tu-darmstadt.de
}

\begin{abstract}
Existing approaches to active learning maximize the system performance by sampling unlabeled instances for annotation that yield the most efficient training. However, when active learning is integrated with an end-user application, this can lead to frustration for participating users, as they spend time labeling instances that they would not otherwise be interested in reading. In this paper, we propose a new active learning approach that jointly optimizes the seemingly counteracting objectives of the active learning system (training efficiently) and the user (receiving useful instances). We study our approach in an educational application, which particularly benefits from this technique as the system needs to rapidly learn to predict the appropriateness of an exercise to a particular user, while the users should receive only exercises that match their skills. We evaluate multiple learning strategies and user types with data from real users and find that our joint approach better satisfies both objectives when alternative methods lead to many unsuitable exercises for end users. ${ }^{1}$
\end{abstract}

\section{Introduction}

State-of-the-art machine learning approaches require huge amounts of training data. But for many NLP applications, there is little to no training data available. Interactive NLP systems are a viable solution to alleviate the cost of creating large training datasets before a new application can be used. Such systems start with no or few labeled instances and acquire additional training data based on user feedback for their predictions. Active learning (Settles, 2012) is a frequently used technique to quickly maximize the prediction performance, as the system acquires user feedback in each iteration for

\footnotetext{
${ }^{1}$ Our code and simulated learner models are available on Github: https://github.com/UKPLab/ acl2020-empowering-active-learning
}

those instances that likely yield the highest performance improvement (e.g., because the system is yet uncertain about them). Active learning has been shown to reduce the amount of user feedback required while improving system performance for interactive NLP systems (P.V.S and Meyer, 2017; Gao et al., 2018) and to reduce the annotation costs in crowdsourcing scenarios (Fang et al., 2014). However, outside the typical annotation setup, it can be boring or frustrating for users to provide feedback on ill-predicted instances that hardly solve their needs. Consider a newly launched web application for learning a foreign language, which aims at suggesting exercises that match the user's proficiency according to Vygotsky's Zone of proximal development (Vygotsky, 1978). The underlying machine learning system starts without any data, but employs active learning to select an exercise the system cannot confidently predict. Then, it adjusts its model interactively based on the user's feedback. While the system is still uncertain, the users often receive inappropriate (e.g., too hard or too easy) exercises. Thus, they get the impression that the system does not work properly, which is especially harmful during the inception phase of an application, as the community opinion largely defines its success.

In this paper, we distinguish the system objective of maximizing the prediction performance with minimal labeled instances and the user objective of providing useful instances for the user's current needs. For the first time, we propose an active learning approach that jointly optimizes these seemingly counteracting objectives and thus trades off the demands of system and user.

The users of educational applications can particularly benefit from this, as they can learn most if they receive appropriate learning material while the underlying system requires considerable training to reach acceptable performance. We employ our 


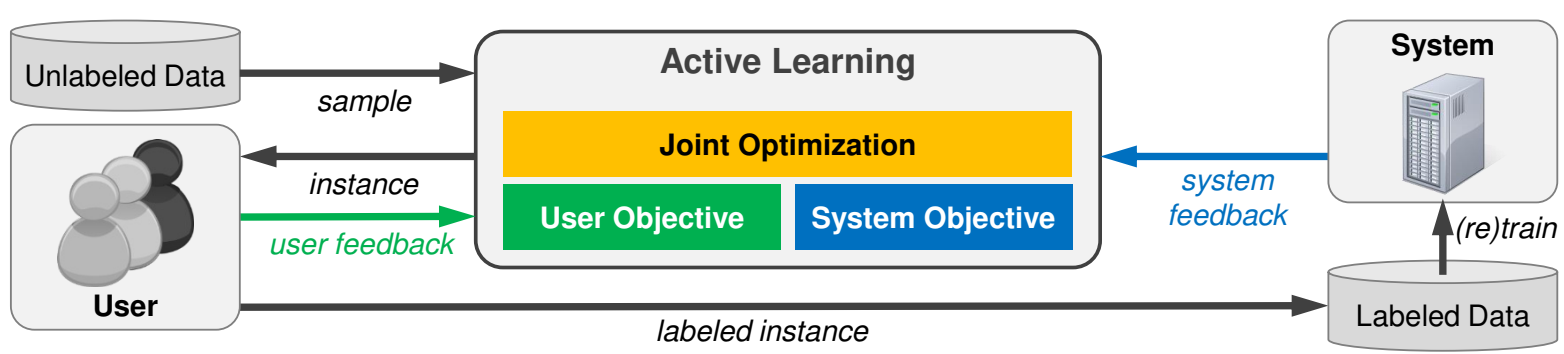

Figure 1: Overview of our interactive approach. We go beyond previous work on optimizing the system objective (blue) by modeling the user objective (green) and jointly optimizing these seemingly counteracting goals (gold).

new approach in a language learning platform for C-tests (i.e., cloze tests, in which the second half of every second word is replaced by a gap). Our system successfully learns how to predict the difficulty of a C-test gap (system objective) and how to provide a C-test that is neither too easy for the current user, which would cause boredom, nor too hard, which would create frustration (user objective). Predicting the difficulty of an exercise and correspondingly selecting exercises that match a user's proficiency are important steps towards selfdirected language learning and massive open online courses (MOOCs) on language learning. Though we focus on this educational use case in this paper, our approach may also yield new insights for other problems that suffer from seemingly counteracting system and user objectives, for example, interactively trained recommender systems for books, movies, or restaurants.

\section{Related Work}

Active learning. Active learning aims to reduce the amount of training data by intelligently sampling instances that benefit the model most (Settles, 2012). A distinct characteristic of active learning is that labels for sampled instances are unknown and provided by an oracle after sampling. Various works investigate the use of active learning for crowdsourcing, where the oracles (i.e., the crowdworkers) may provide noisy labels (Snow et al., 2008; Laws et al., 2011). Within the educational domain, active learning research is scarce. ${ }^{2}$ One example is the work by Rastogi et al. (2018), who propose a threshold-based sampling strategy utilizing the prediction probability and achieve a considerable speed-up without any significant performance drop. Hastings et al. (2018) find that ac-

\footnotetext{
${ }^{2}$ Note, that in education, active learning often refers to a teaching paradigm which is unrelated to active learning in machine learning.
}

tive learning can be used to efficiently train a system for providing feedback on student essays using teachers as oracles. Horbach and Palmer (2016) report mixed results for employing active learning in short-answer grading. While all of these works focus on improvements of the proposed system, users only benefit after training. In contrast, our work explicitly models the user objective, such that users already benefit while labeling training instances.

Adaptive learning. Many systems provide user adaptation, and research has shifted from predefined sets of rules for adaptation to data-driven approaches. Several works investigate adaptive methods to provide exercises which are neither too hard nor too boring. For instance, Missura and Gärtner (2011) model learning in a game-theoretic sense where the goal is to adjust the difficulty to neither being too easy nor too hard. Other works investigate adaptation in the context of testing (Zheng and Chang, 2015; Wang et al., 2016; Chaimongkol et al., 2016) and propose methods for an adaptive selection of appropriate tests for better assessing a student's proficiency. In a large survey, Truong (2016) discusses how to integrate different learning styles, modeling categorical student behavior, into an adaptive learning environment and emphasizes the need for more sophisticated methods.

Despite much research in adaptive and active learning, none of the previous works consider jointly modeling and optimizing both the system and user objectives which may retain a user's motivation and keep them from leaving the platform due to boredom or frustration.

\section{Approach}

Figure 1 shows our proposed interactive learning setup. The active learning component iteratively samples instances from a pool of unlabeled data and asks the user for a label that can be used to 
train the machine learning system. Previous work on active learning focused on optimizing the system objective (blue). That is, only the system provides feedback to the active learning component (e.g., how certain it is about the predicted label of an instance). In our work, we first model the user objective (green) and propose sampling strategies that maximize the user satisfaction based on the user's feedback (e.g., the user's label for an instance). Finally, we study our novel joint optimization strategies (gold) that trade off the demands of the system and the users. Whereas we distinguish between the user's feedback (exercise-level) and labeled instances (gap-level) in our work, our proposed approach can easily be adapted to more specific cases where the (implicit) user feedback and the provided label are the same. ${ }^{3}$

In the remainder of this section, we introduce sampling strategies that select which instance should be presented to the user next. We use the following notation: Let $\mathcal{X}$ be the pool of unlabeled instances. In every iteration of the application (e.g., when a user requests a new exercise), the sampling strategy $s(v)$ returns an instance $x \in \mathcal{X}$ for user $v$. The user then provides a label $y$ for instance $x$, potentially with additional feedback on the user's satisfaction. The active learning component finally removes $x$ from its pool $\mathcal{X}$ and adds $(x, y)$ to the set of labeled instances, before the system is retrained with the increased labeled training set.

The simplest sampling strategy that we use as a baseline is random sampling $s_{\text {rand }}(v)$, which selects an $x \in \mathcal{X}$ uniformly at random, regardless of the user. In the following subsections, we discuss more advanced strategies that optimize the system or user objective as well as our new joint optimization strategies.

\subsection{System optimization}

To optimize the system objective, we consider uncertainty sampling (Lewis and Gale, 1994). Uncertainty sampling assumes that instances for which the model is least certain during prediction provide the most information for the model once their labels are known. The sampled instance is thus

$$
s_{\text {unc }}(v)=\arg \max _{x \in \mathcal{X}} U(x)
$$

\footnotetext{
${ }^{3}$ Note, that from a single answer which is either correct or wrong, we cannot deduce a fine-grained gap label. To obtain these in a real-world setting, one either may assume querying groups of users or asking them for an explicit label.
}

where $U: x \mapsto[0,1]$ returns the uncertainty of predicting a label for instance $x$. Like random sampling, $s_{\text {unc }}(v)$ is independent of the current user $v$. A model's uncertainty can be measured in multiple different ways, for example, by the prediction probability of the predicted label (Lewis and Gale, 1994), as the difference in probabilities between the first and second most probable labels (Scheffer et al., 2001), and based on the Shannon entropy (Shannon, 1948) that considers all possible labels (Settles and Craven, 2008). We instantiate $U$ for our educational application in section 4 .

\subsection{User optimization}

The objective of users is to receive instances that meet their demands. We therefore define a new user-oriented sampling strategy as

$$
s_{\mathrm{usr}}(v)=\arg \max _{x \in \mathcal{X}} A(x, v)
$$

where $A:(x, v) \mapsto[0,1]$ returns the degree of appropriateness of instance $x$ for the user $v$. In our educational application, we consider an exercise appropriate if it is neither too easy nor too difficult, as this maximizes the user's learning gain. To quantify $A$, we measure the error between the predicted label $f(x)$ and the user's demand $\phi(v)$ as

$$
A(x, v)=1-\operatorname{err}[f(x), \phi(v)]
$$

with an error function err $\in[0,1]$ (cf., section 4).

\subsection{Joint optimization}

We propose two novel strategies to jointly optimize the user and system objectives.

Combined sampling. Our first strategy

$$
s_{\text {comb }}(v)=\arg \max _{x \in \mathcal{X}} U(x) A(x, v)
$$

combines uncertainty sampling and user-oriented sampling by preferring appropriate instances for user $v$ (as in $s_{\text {usr }}$ ), but among them returns the one the system is most uncertain about (as in $s_{\text {unc }}$ ).

Trade-off sampling. For our second strategy, we aggregate both objectives into a single function

$$
\begin{aligned}
s_{\text {tos }}(v)=\arg \max _{x \in \mathcal{X}}\{(1-\lambda) A(x, v) \\
+\lambda U(x)\}
\end{aligned}
$$

which is the weighted sum of user-oriented and uncertainty sampling. The weight parameter $\lambda \epsilon$ $[0,1]$ can be used to adjust the learning towards the system objective or the user objective. 


\section{Instantiation}

We consider our jointly optimized active learning particularly beneficial for educational applications, since (1) the users of such a system may fail to achieve their learning goals with inappropriate exercises. Additionally, (2) it is difficult to acquire large difficulty-annotated datasets for training, as actual users are required for producing realistic training data and existing learner datasets can hardly be shared due to privacy concerns. We therefore instantiate our approach for a language learning platform that predicts the difficulty of exercises and learns to provide appropriate (neither too easy nor too hard) exercises to its users.

C-tests. For our experiments, we use the setup of the C-test difficulty prediction task as investigated by Beinborn (2016). C-tests are gap filling exercises proposed by Klein-Braley and Raatz (1982). In their proposed gap scheme, every second word is turned into a gap by removing the latter half of its characters. In contrast to cloze tests, C-tests do not require any distractors, since the first half of the word remains as a hint. Solving $\mathrm{C}$-tests requires orthographic, morphologic, syntactic, and semantic competencies as well as general vocabulary knowledge (Chapelle, 1994). C-tests can be easily created automatically by choosing an arbitrary text and introducing the gaps as described above. Because of the context and the kept word prefixes, C-test gaps typically only allow for a single solution (given by the original text) and therefore do not require manual correction. The biggest challenge, however, lies in controlling the difficulty of the text and the derived C-test with its gaps as we have shown in previous work (Lee et al., 2019).

System objective. Given a large pool $\mathcal{X}$ of Ctests $x \in \mathcal{X}$ with $n$ gaps $g_{i} \in x, 1 \leq i \leq n$, the system objective is to learn a classifier $d(g) \in$ $L_{D}$ to judge the gap difficulty of gaps $g \in x$ with minimal training data. As the difficulty classes $L_{D}$, we use the four labels very easy, easy, hard, and very hard proposed by Beinborn (2016). These four classes are based on the mean error rates $e(g)$ of a gap $g$ observed across all users. Figure 2 shows the mapping between the mean error rates $e(g)$ and the four gap difficulty classes $L_{D}$.

Data. For our experiments, we obtained 3,408 solutions to English C-tests from our university's language center. Each participant solved five C-

\begin{tabular}{c|c|c|c} 
very easy & easy & hard & very hard \\
\hline$[0,0.25[$ & {$[0.25,0.5[$} & {$[0.5,0.75[$} & {$[0.75,1]$}
\end{tabular}

Figure 2: Gap difficulty classes and error rate ranges

tests with 20 gaps each (i.e., 100 gaps per solution). The five C-tests vary across the participants based on a set of 74 different $\mathrm{C}$-tests in total. We filter out answers from 22 participants who either did not provide any correct answer or only filled out the first of the five C-tests. Based on this dataset, we derive the ground-truth labels for the gap difficulty classification $d(g)$ based on figure 2 .

Aggregated instances. In contrast to Beinborn's (2016) work, a particular challenge of our setup is the need to aggregate instances. The active learning strategies $s(v)$ always sample entire Ctests $x \in \mathcal{X}$ and judge their appropriateness for a user $v$ based on $A(x, v)$. The underlying classifier $d(g)$, however, operates at the level of gaps $g \in x$ within a C-test. Similarly complex setups can be found in multiple other real-world tasks, including educational applications (e.g., providing reading recommendations at book or chapter level, but estimating appropriateness at word or sentence level) and product recommendation tasks (e.g., training a classifier for cast, plot, and action aspects, but recommending entire movies).

For our instantiation, we measure the classifier's uncertainty using the Shannon entropy

$$
H(g)=-\sum_{\ell \in L_{D}} P(\ell \mid g) \log P(\ell \mid g)
$$

across the four difficulty classes $L_{D}$ of a gap $g$. $P(\ell \mid g)$ denotes the probability of the classifier $d$ to assign the difficulty class $\ell$ to gap $g$. We then aggregate the resulting scores similar to the total token entropy proposed by Settles and Craven (2008):

$$
U_{\text {ent }}(x)=\frac{1}{n} \sum_{i=1}^{n} \frac{H\left(g_{i}\right)}{H_{\max }}
$$

where $H_{\max }$ is the maximum achievable Shannon entropy, which serves as a normalization term. $H_{\text {max }}$ can be pre-computed as:

$$
H_{\max }=-\sum_{i=1}^{\left|L_{D}\right|} \frac{1}{\left|L_{D}\right|} \log \frac{1}{\left|L_{D}\right|}
$$

User objective. To model the demands of the users, we define five proficiency levels $L_{P}=$ 


\begin{tabular}{lccccc}
\hline Level & 1 & 2 & 3 & 4 & 5 \\
\hline Score (\%) & $0-54$ & $55-64$ & $65-74$ & $75-84$ & $85-100$ \\
Users & 814 & 607 & 724 & 769 & 472 \\
\hline
\end{tabular}

Table 1: Proficiency levels, corresponding scores (\% correctly filled gaps), and number of users per level.

$\{1,2,3,4,5\}$ based on the users' ability to solve C-tests. The user representation $\phi(v) \in L_{P}$ of user $v$ thus returns a proficiency level between 1 and 5 with 5 indicating the highest proficiency.

In our experiments, we use the C-test dataset introduced above to obtain $\phi(v)$. Note that in this dataset, each user solved exactly five C-tests. We therefore map their score (i.e., the percentage of correctly filled gaps) to a proficiency level that roughly corresponds to the language courses offered by the university language center. Table 1 shows the five levels with their corresponding score ranges and the number of users in the dataset.

We estimate the proficiency level of a C-test $x=g_{1}, g_{2}, \ldots, g_{n}$ with

$$
f(x)=\psi\left(\frac{1}{n} \sum_{i=1}^{n} c\left(g_{i}\right)\right)
$$

where $c: g \mapsto\{0,1\}$ is an indicator function to predict if gap $g_{i}$ will be correctly (1) or incorrectly (0) answered and $\psi$ maps the percentage of correct answers to the corresponding proficiency level according to Table 1. For our experiments, we define

$$
c(g)= \begin{cases}1 & \text { if } k<j \\ 0 & \text { otherwise }\end{cases}
$$

where $k \sim \mathcal{U}\left(\frac{\ell-1}{\mid L_{P}}, \frac{\ell}{\left|L_{P}\right|}\right)$ and $j \sim \mathcal{U}(0,1)$ are uniformly sampled random variables and $\ell=d(g)$. Based on our estimation $f(x) \in L_{P}$, we can now define the error function err as the normalized distance of $f(x)$ to the required proficiency:

$$
\operatorname{err}[f(x), \phi(v)]=\frac{1}{\left|L_{P}\right|}|f(x)-\phi(v)|
$$

\section{Experimental Setup}

System setup. We initialize our system with an empty set of labeled instances. In every iteration, we sample a C-test consisting of 20 gaps from the pool of unlabeled instances $\mathcal{X}$ using one of the sampling strategies introduced in the previous section. Then, we obtain labels based on how the user solved the test, which contributes (1) to the overall difficulty prediction for each gap and (2) to the representation of the current user's proficiency.
Our approach can be used with any underlying classifier $d(g)$. In this paper, we train a multilayer perceptron (MLP) to predict the four difficulty classes for a C-test gap. To represent the input of the MLP, we use the 59 features previously proposed by Beinborn (2016). We furthermore introduce two novel features computed from BERT (Devlin et al., 2019): We hypothesize that the masking objective of BERT which masks individual words during training is very similar to a gap filling exercise and thus, a model trained in such a way may provide useful signals for assessing the difficulty of a gap. For each gap, we generate a sentence where only the gap is replaced by the masking token and fetch its predictions from the BERT model. From these predictions we take the prediction probability of the solution as the first feature and the entropy of the prediction probabilities of the top-50 predicted words as the second feature in concordance with findings by Felice and Buttery (2019) who show that entropy strongly correlates with the gap difficulty. Adding both features to the 59 features proposed by Beinborn (2016) increases the accuracy of our MLP from 0.33 to $0.37 .^{4}$

While Beinborn successfully used support vector machines (SVM) in her work, we find that MLPs perform on par with SVMs (for the old and new features) and that they are more robust regarding the choice of the first sampled instance. Moreover, in our initial experiments with little training data, SVMs and Logistic Regression classifiers were only able to predict the majority class.

Our MLP has a single hidden layer consisting of 61 hidden units. We train the neural network for 250 epochs with early stopping after 20 epochs without any improvement and use Adam (Kingma and $\mathrm{Ba}, 2015$ ) as our optimizer. Note that our main interest is in the analysis of the novel active learning approach, which is why we do not systematically study the underlying classifier, but use a setup comparable to the state-of-the-art results reported by Beinborn (2016).

We run experiments for each of our sampling strategy. We select five C-tests without any overlap between users, texts, and their corresponding user answers to create an independent test set and put the remaining $69 \mathrm{C}$-tests into the pool of unlabeled data. In the first iteration, we use the randomly initialized weights of our neural network to select

\footnotetext{
${ }^{4}$ The results are averaged across ten runs with different random initializations.
} 
the starting example. To provide comparable results between different runs, we keep the parameter initialization of our neural network fixed when comparing different sampling strategies. We limit each experimental run to $8 \cdot 5=40$ iterations, as the five proficiency levels are not evenly distributed with the smallest class having only eight C-tests. At each iteration, we train our model on $80 \%$ of the already labeled data and use the remaining $20 \%$ as our validation set (split randomly). We use the bestperforming model on the validation set for testing and store it as our model initialization for the next iteration. On an Intel Core i5-4590, a single run with 40 iterations takes less than four minutes.

Learner behavior. To study the benefit of our approach for different types of learners, ${ }^{5}$ we derive four prototypical learner behaviors from our $\mathrm{C}$-test dataset. To prepare this, we first compile a probabilistic model for the learners of each proficiency group as described in Table 1 to obtain learner-specific gap error rates $e(g, v)$. The learnerspecific gap error rates are computed by binning all learners into the specific groups and then computing the error rate by averaging for each gap. If there is no error rate for a given gap and learner in our dataset, we use the averaged gap error rate of the corresponding proficiency group to simulate an answer.

Using these learner-specific gap error rates, we predict whether an answer to a C-test gap $g$ is correct or incorrect similar to Equation (10):

$$
\hat{c}(g)= \begin{cases}1 & \text { if } e(g, v)<j \\ 0 & \text { otherwise }\end{cases}
$$

In contrast to Equation (10), we do not sample $k$, but use the learner-specific error rates $e(g, v)$ for gap $g_{i}$ from the proficiency level $\phi(v)$. Again, $j \sim$ $\mathcal{U}(0,1)$ is a uniformly sampled random variable.

For a language learning platform, it is likely that motivated learners who continually practice improve their proficiency over time. Less motivated learners or learners who suffer from distractions, interruptions, or frustration, however, may show different paces in their learning speed or even deteriorate in their proficiency. Therefore, we study four prototypical types of learner behavior:

- Static learners (STAT) do not improve their skills over the course of our experiments. Instead, they provide answers constantly at the

\footnotetext{
${ }^{5}$ Henceforth, we use learner to refer to the users of an educational application rather than to a machine learning system.
}

same, pre-defined proficiency level. This models learners with a slow progress or with little motivation overall.

- Motivated learners (MOT) continually improve their language proficiency throughout our experiments with a fixed step size of $t_{1} \mathrm{C}$-tests. That is, we simulate that their proficiency level $\phi(v)$ increases by one every $t_{1}$ iterations.

- Interrupted learners (INT) experience a drop in their proficiency during our experiments. Such cases occur, for example, if a learner has to interrupt their learning process for a longer time. For our simulation, we start with the motivated learner setup, constantly increasing the proficiency every $t_{1}$ iterations. However, this learner experiences a sudden increase $\left(t_{2}\right)$ and drop $\left(t_{3}\right)$ in the proficiency level by one. After recovering from the drop $\left(t_{4}\right)$ the proficiency will again increase according to the motivated learner $\left(t_{5}\right)$.

- Artificially decreasing learner. (DEC) Finally, our last group of simulated learners displays a constant drop in their proficiency during our simulation. Although such cases rarely occur in the real world, we use this learner to evaluate all sampling strategies in the case of constant drop. Similar to the motivated learner, we start with the highest possible proficiency and decrease it by one every $t_{1}$ iterations.

For our experiments, we assume a static learner that remains at proficiency level $\phi(v)=3$. For motivated learners, we set the initial proficiency level to 1 and use a step size of $t_{1}=8$, so that they traverse all proficiency levels throughout a single run. For interrupted learners, we also use $t_{1}=8$ with an additional increase after $t_{2}=12$, a drop after $t_{3}=16$, and a recovery (increase) after $t_{4}=20$. Starting from $t_{5}=24$, interrupted learners behave the same as motivated learners.

Like Beinborn (2016), we cannot publish the C-test data due to data privacy reasons, but we provide our code and simulated learner models on GitHub. $^{6}$

\section{Experiments}

We present and discuss our results for $U_{\text {ent }}$ and $A$ as defined in section 4 . For each strategy we run our experiments ten times with different weight

\footnotetext{
${ }^{6}$ https://github.com/UKPLab/ acl2020-empowering-active-learning
} 
initializations and report the averaged scores. For random sampling, we do ten runs with different random seeds for each weight initialization to provide more stable results. We set $\lambda=0.5$ for our trade-off sampling strategy.

\subsection{Evaluation metrics}

As our system and user objectives have different scopes (gap-level vs. exercise-level), we quantify both differently. To measure the system objective, we report the accuracy of our model for predicting the individual gap difficulties of the test data after each iteration. As our training data increases by 20 gaps after each iteration, we provide plots for all experiments from the first to the last (40th) iteration. For quantifying the user objective, we evaluate all sampling strategies across all 40 iterations, i.e., how well our sampling strategies were able to satisfy the user's needs after the whole set of exercises. Instead of accuracy, we take the distance-based metric mean absolute error (MAE). As users explicitly query a C-test of a specific proficiency level at each iteration, suggesting a $\mathrm{C}$-test which deviates by two levels from the requested proficiency has a worse impact on the user's learning experience than a C-test which only deviates by one level. For better interpretability, we do not normalize the MAE as we do for our error function err, i.e., a MAE of 1 means that on average, the difficulty of the sampled instances was off by a whole proficiency level from the queried ones.

\subsection{Results}

Since the interrupted learner experiences both a drop and increase in proficiency in a less constant manner than the motivated or decreasing learners, we conduct further analysis of our sampling strategies for the interrupted learner.

System objective. Figure 3 shows the system objective for $U_{\text {ent }}$ after each iteration. Vertical blue lines indicate increases in the learner's proficiency whereas the vertical yellow line indicates a drop. We observe that although random sampling performs rather well in the early iterations, all our proposed strategies as well as the uncertainty sampling baseline are able to outperform it in the later iterations. Moreover, all proposed strategies perform similar to uncertainty sampling. This is surprising, especially for the user-oriented sampling strategy as it inherently does not optimize the system objective. One reason for this may be the similarity

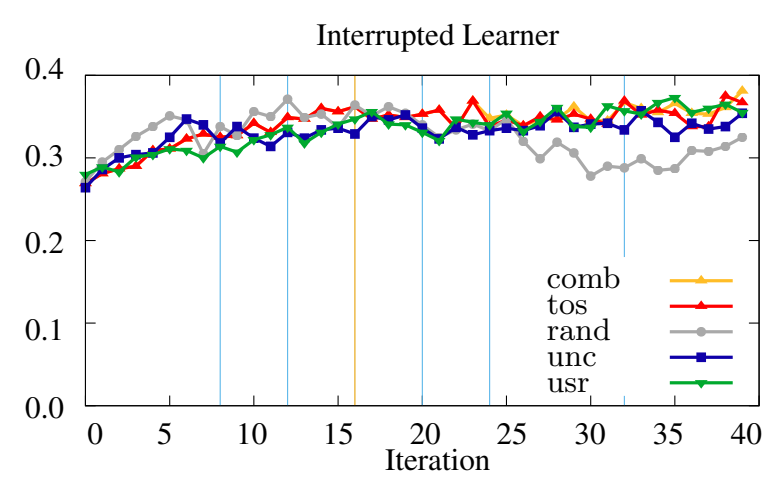

Figure 3: Accuracy on the test data for $U_{\mathrm{ent}}$.

\begin{tabular}{lcccc}
\hline & STAT & MOT & INT & DEC \\
\hline tos & $\mathbf{. 3 4 4}$ & .338 & .339 & .327 \\
comb & .343 & $\mathbf{. 3 4 0}$ & $\mathbf{. 3 4 1}$ & .327 \\
usr & .338 & .331 & .334 & .328 \\
unc & .332 & .331 & .331 & $\mathbf{. 3 3 1}$ \\
rand & .325 & .325 & .325 & .325 \\
\hline
\end{tabular}

Table 2: Averaged accuracy over all iterations for $U_{\text {ent }}$

of the user-oriented sampling strategy to curriculum learning (Bengio et al., 2009), which opts to organize model training in a meaningful way. As we sample instances the model is most confident in (i.e., have the highest prediction confidence) this leads to instances which are easier to learn and may especially be helpful in low-data scenarios.

To better quantify our results, we compare the averaged accuracy scores across all iterations, shown in table 2 and conduct Wilcoxon signed-rank tests (Wilcoxon, 1992) on the active learning curves for system and model objectives to test for statistical significance. We can observe that for the static, motivated, and interrupted learners both our joint sampling strategies outperform all baselines significantly $(\mathrm{p}<0.05)$, but show no significant difference between each other. ${ }^{7}$ Only for the decreasing learner all strategies show no significant difference at all. In concordance with our observations for the user-oriented sampling which may benefit from first sampling easy-to-learn instances, jointly optimizing system and user objective seems to benefit from curriculum learning and active learning paradigms.

User objective. Table 3 shows the MAE for all strategies using $U_{\text {ent }}$. We can observe that all strate-

\footnotetext{
${ }^{7}$ The system performance of random sampling remains the same for all learner types as it is averaged across all runs.
} 


\begin{tabular}{lcccc}
\hline & STAT & MOT & INT & DEC \\
\hline tos & 0.98 & 0.65 & 0.93 & 0.75 \\
comb & 0.98 & 0.63 & 0.88 & $\mathbf{0 . 6 5}$ \\
usr & $\mathbf{0 . 8 5}$ & $\mathbf{0 . 5 8}$ & $\mathbf{0 . 6 5}$ & 0.75 \\
unc & 1.17 & 1.33 & 1.35 & 1.72 \\
rand & 1.16 & 1.22 & 1.82 & 1.24 \\
\hline
\end{tabular}

Table 3: MAE for $U_{\text {ent }}$

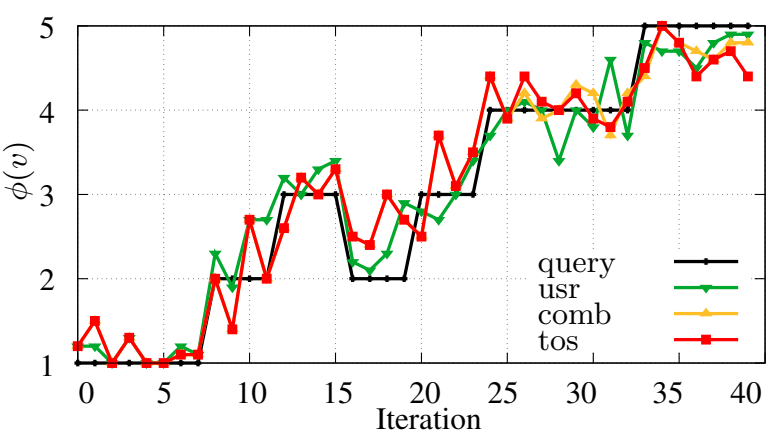

Figure 4: Sampled instances for the interrupted learner.

gies which consider a separate user objective sample instances which significantly better fit the current user proficiency. ${ }^{8}$ Furthermore, the combined sampling approach which puts more emphasis on the user objective outperforms our trade-off sampling for all learner behaviors and even manages to outperform the user-oriented sampling strategy for the decreasing learner.

We further investigate how well our approaches react to changes in the user objective by plotting the mean difficulty $\phi(v)$ of sampled instances after each step for all our strategies modeling the user objective. As figure 4 shows, all sampling strategies are able to match the queried C-test difficulties well, as they do not deviate much from the queried difficulty (in black).

Adaptive choice of $\lambda$. We furthermore investigate how the choice of $\lambda$ affects our trade-off sampling strategy. As the system predictions may not be very accurate in early iterations, it is reasonable to put more emphasis on the system objective in the beginning, but focus on providing suited C-tests (user objective) in later iterations. We thus define $\lambda$ as an adaptive function $\lambda=f(i)=\frac{1}{\sqrt{i}}=i^{-0.5}$ which highly emphasizes the system objective in early stages and anneals with an increasing number

\footnotetext{
${ }^{8}$ Statistical testing was again conducted using a Wilcoxon signed-rank test for $\mathrm{p}<0.05$.
}

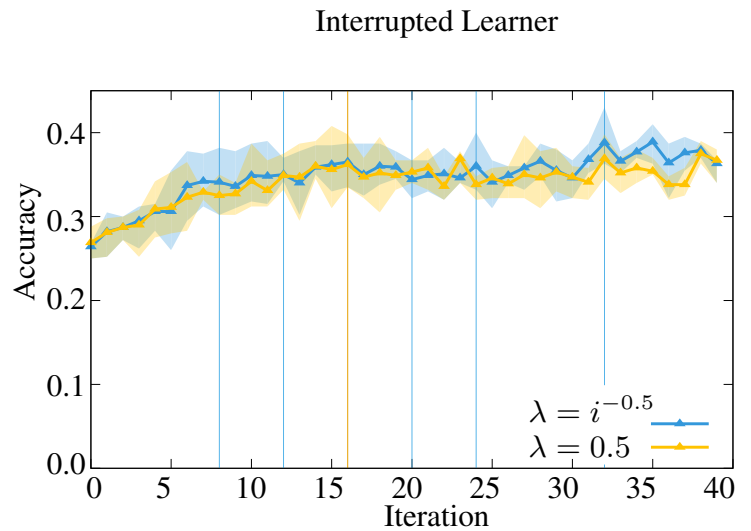

Figure 5: Accuracy of tos for annealed and fixed $\lambda$.

\begin{tabular}{lcccc}
\hline Acc & STAT & MOT & INT & DEC \\
\hline $\operatorname{tos}_{\lambda}$ & .333 & $\mathbf{. 3 4 6}$ & $\mathbf{. 3 4 7}$ & .314 \\
$\operatorname{tos}$ & .334 & .338 & .339 & .327 \\
\hline MAE & STAT & MOT & INT & DEC \\
\hline $\operatorname{tos}_{\lambda}$ & $\mathbf{0 . 8 5}$ & $\mathbf{0 . 5 3}$ & $\mathbf{0 . 4 8}$ & $\mathbf{0 . 5 3}$ \\
$\operatorname{tos}$ & 0.98 & 0.65 & 0.93 & 0.75 \\
\hline
\end{tabular}

Table 4: Averaged accuracy scores and MAE with an annealed $\lambda$ for $U_{\text {ent }}$.

of iterations $i$.

Figure 5 shows the system performance of our trade-off sampling strategy averaged across ten different runs. The colored areas show the corresponding upper and lower quartiles. As shown in table 4, we can see that our annealed $\lambda$ leads to considerable improvements for system and user objective, leading to a significant increase in average accuracy from 0.339 to 0.347 and a decrease in the MAE from 0.93 to 0.48 for the interrupted learner, outperforming all other sampling strategies.

Further findings. We observe similar results for system and user objectives for the other learner types. Investigating the stability of all sampling approaches furthermore shows that our joint optimization strategies perform better and more stable in early iterations.

Due to averaging, $U_{\text {ent }}$ cannot distinguish between C-tests with only a few highly uncertain gaps and C-tests which have a higher number of less uncertain gaps. However, in preliminary experiments with a different aggregation function which is more robust to C-tests with only a few highly uncertain gaps, we come to similar findings across all sampling strategies and learner types. Detailed results for our other learner behaviors, the stability of our sampling strategies, and the results of our pre- 
liminary experiments with a different aggregation function are provided in the paper's appendix.

Limitations. Although our setup with simulated learners may seem artificial compared to an evaluation study with real-world learners, to conduct such a study in an ethical way, we need to ensure that participants are not hurt in their learning process. Thus, strategies which can be evaluated in user studies are limited to those which consider the user objective. In contrast, the use of simulated learners allows us to compare our proposed strategies against common active learning strategies which do not consider the user objective at all.

Another limitation is how to estimate a learner's current proficiency given that we do not know the true difficulty of a C-test. This raises the general question of using relative or absolute difficulties for the selection of suited exercises. In this work, we assumed absolute proficiency levels and implemented according learner behaviors to provide a more controlled environment for our experiments. In the case of absence of any absolute (true) difficulty estimations for C-tests, we see several directions for future work:

a) As a simple baseline, a normalized version of $\psi(x)$ may be applied on a learner's previously filled-out C-tests. However, this assumes that all C-tests are equally difficult which may lead to unsuited C-tests.

b) Training an additional model for assessing a learner's proficiency given their results on a C-test with the gap-difficulty predictions from our model serving as additional input.

c) Instead of using the absolute difficulty, one may define an optimal error margin as a zone of proximal development (Vygotsky, 1978). This requires an adaptation of the user objective to the relative difficulties of exercises for individual learners, but may be an important step in achieving highly personalized user models without any absolute labels.

\section{Conclusion}

In this work, we investigated how we can incorporate user feedback into existing active learning approaches without hurting the user's actual needs. We formalize both system (active learning) and user objectives and propose two novel sampling strategies which aim to maximize both objectives jointly.
We evaluate our sampling strategies for the task of selecting suited C-tests, a type of fill-the-gap exercise, which fit the current proficiency of a human learner. We create simulated learners for five different proficiency levels from real-world data and use them to define different learning behaviors. Our experiments show that both our novel sampling strategies are successfully selecting instances which lead to a better model training while not hurting a learner's progress by selecting too easy or too difficult C-tests. Although system and user objective at first seem counteracting, our experiments indicate that they complement each other as jointly optimizing them outperforms optimizing only one of the goals. Additional experiments with an adaptive $\lambda$ for our trade-off sampling strategy show that properly balancing system and user objective can lead to considerable improvements in performance for both objectives.

Our findings open up new opportunities for training models on low-resource scenarios with implicitly collected user feedback while jointly serving the user's actual needs. Additional use cases like the training of personalized recommendation models as well as the use of reinforcement learning to find a good trade-off between system and user objective remain to be investigated in future work.

\section{Acknowledgments}

This work has been supported by the German Research Foundation with the ArguAna project (GU 798/20-1) and the Evidence project (GU 798/27-1). We thank the anonymous reviewers for their detailed and helpful comments as well as Edwin Simpson and Yevgeniy Puzikov for the insightful discussions about our work. We especially thank the language center of the Technische Universität Darmstadt for providing us with the data and Dr. Lisa Beinborn for providing us with the code to extract her proposed features.

\section{References}

Lisa Marina Beinborn. 2016. Predicting and Manipulating the Difficulty of Text-Completion Exercises for Language Learning. Ph.D. thesis, Technische Universität Darmstadt, Darmstadt.

Yoshua Bengio, Jérôme Louradour, Ronan Collobert, and Jason Weston. 2009. Curriculum learning. In Proceedings of the 26th Annual International Conference on Machine Learning, ICML '09, pages 4148, New York, NY, USA. ACM. 
Nhabhat Chaimongkol, Shotiga Pasiphol, and Sirichai Kanjanawasee. 2016. Computerized Adaptive Testing with Reflective Feedback: A Conceptual Framework. Procedia-Social and Behavioral Sciences, 217:806-812.

C. A. Chapelle. 1994. Are C-tests valid measures for L2 vocabulary research? Second Language Research, 10(2):157-187.

Jacob Devlin, Ming-Wei Chang, Kenton Lee, and Kristina Toutanova. 2019. BERT: Pre-training of deep bidirectional transformers for language understanding. In Proceedings of the 2019 Conference of the North American Chapter of the Association for Computational Linguistics: Human Language Technologies (NAACL/HLT), pages 4171-4186, Minneapolis, MI, USA.

Meng Fang, Jie Yin, and Dacheng Tao. 2014. Active learning for crowdsourcing using knowledge transfer. In Proceedings of the Twenty-Eighth AAAI Conference on Artificial Intelligence (AAAI), pages 1809-1815, Qubec, Canada.

Mariano Felice and Paula Buttery. 2019. Entropy as a proxy for gap complexity in open cloze tests. In Proceedings of the International Conference Recent Advances in Natural Language Processing (RANLP), pages 323-327, Varna, Bulgaria.

Yang Gao, Christian M Meyer, and Iryna Gurevych. 2018. APRIL: Interactively Learning to Summarise by Combining Active Preference Learning and Reinforcement Learning. In Proceedings of the 2018 Conference on Empirical Methods in Natural Language Processing, pages 4120-4130, Brussels, Belgium.

Peter Hastings, Simon Hughes, and M. Anne Britt. 2018. Active learning for improving machine learning of student explanatory essays. In Carolyn Penstein Rosé, Roberto Martnez-Maldonado, H. Ulrich Hoppe, Rose Luckin, Manolis Mavrikis, Kaska Porayska-Pomsta, Bruce McLaren, and Benedict du Boulay, editors, Artificial Intelligence in Education (AIED), volume 10947 of Lecture Notes in Computer Science, pages 140-153. Cham: Springer.

Andrea Horbach and Alexis Palmer. 2016. Investigating Active Learning for Short-Answer Scoring. In Proceedings of the 11th Workshop on Innovative Use of NLP for Building Educational Applications (BEA), pages 301-311, San Diego, CA, USA.

Diederik P. Kingma and Jimmy Lei Ba. 2015. Adam: A method for stochastic optimization. In Proceedings of the 3rd International Conference on Learning Representations (ICLR), San Diego, CA, USA.

Christine Klein-Braley and Ulrich Raatz. 1982. Der C-Test: ein neuer Ansatz zur Messung allgemeiner Sprachbeherrschung. AKS-Rundbrief, 4:23-37.
Florian Laws, Christian Scheible, and Hinrich Schütze. 2011. Active Learning with Amazon Mechanical Turk. In Proceedings of the Conference on Empirical Methods in Natural Language Processing (EMNLP), pages 1546-1556, Edinburgh, UK.

Ji-Ung Lee, Erik Schwan, and Christian M. Meyer. 2019. Manipulating the difficulty of c-tests. In Proceedings of the 57th Annual Meeting of the Association for Computational Linguistics, pages 360-370, Florence, Italy.

David D. Lewis and William A. Gale. 1994. A sequential algorithm for training text classifiers. In Proceedings of the 17th Annual International ACM Conference on Research and Development in Information Retrieval (SIGIR), pages 3-12, Dublin, Ireland.

Olana Missura and Thomas Gärtner. 2011. Predicting dynamic difficulty. In J. Shawe-Taylor, R. S. Zemel, P. L. Bartlett, F. Pereira, and K. Q. Weinberger, editors, Advances in Neural Information Processing Systems 24, pages 2007-2015. Curran Associates.

Avinesh P.V.S and Christian M. Meyer. 2017. Joint optimization of user-desired content in multidocument summaries by learning from user feedback. In Proceedings of the 55th Annual Meeting of the Association for Computational Linguistics (ACL), pages 1353-1363, Vancouver, Canada.

Ishan Rastogi, Aditya Kanade, and Shirish Shevade. 2018. Active learning for efficient testing of student programs. In Carolyn Penstein Rosé, Roberto Martnez-Maldonado, H. Ulrich Hoppe, Rose Luckin, Manolis Mavrikis, Kaska PorayskaPomsta, Bruce McLaren, and Benedict du Boulay, editors, Artificial Intelligence in Education (AIED), volume 10947 of Lecture Notes in Computer Science, pages 296-300. Cham: Springer.

Tobias Scheffer, Christian Decomain, and Stefan Wrobel. 2001. Active Hidden Markov Models for Information Extraction. In Frank Hoffmann, David J. Hand, Niall Adams, Douglas Fisher, and Gabriela Guimaraes, editors, Advances in Intelligent Data Analysis (IDA), volume 2189 of Lecture Notes in Computer Science, pages 309-318. Berlin/Heidelberg: Springer.

Burr Settles. 2012. Active Learning, volume 18 of Synthesis Lectures on Artificial Intelligence and Machine Learning. Morgan \& Claypool.

Burr Settles and Mark Craven. 2008. An Analysis of Active Learning Strategies for Sequence Labeling Tasks. In Proceedings of the Conference on Empirical Methods in Natural Language Processing (EMNLP), pages 1070-1079, Honolulu, HI, USA.

Claude Elwood Shannon. 1948. A mathematical theory of communication. Bell system technical journal, 27(3):379-423. 
Rion Snow, Brendan O'Connor, Daniel Jurafsky, and Andrew Y Ng. 2008. Cheap and Fast - But is it Good? Evaluating Non-Expert Annotations for Natural Language Tasks. In Proceedings of the Conference on Empirical Methods in Natural Language Processing (EMNLP), pages 254-263, Honolulu, HI, USA.

Huong May Truong. 2016. Integrating learning styles and adaptive e-learning system: Current developments, problems and opportunities. Computers in human behavior, 55(Part B):1185-1193.

Lev Vygotsky. 1978. Mind in society: The development of higher psychological processes. Cambridge: Harvard University Press.

Shiyu Wang, Haiyan Lin, Hua-Hua Chang, and Jeff Douglas. 2016. Hybrid Computerized Adaptive Testing: From Group Sequential Design to Fully Sequential Design. Journal of Educational Measurement, 53(1):45-62.

Frank Wilcoxon. 1992. Individual comparisons by ranking methods. In Breakthroughs in statistics, pages 196-202. Springer.

Yi Zheng and Hua-Hua Chang. 2015. On-the-fly assembled multistage adaptive testing. Applied Psychological Measurement, 39(2):104-118.

\section{A Appendices}

\section{A.1 Results of $U_{\text {ent }}$ for other learner types}

Figure 6 shows our results for the static, motivated, and artificially decreasing learner. As with the interrupted learner, blue (yellow) vertical lines indicate an increase (drop) in the learner's proficiency. Similar to the results for the interrupted learner, all strategies outperform random sampling in later iterations.

\section{A.2 An outlier-invariant variation of $U$}

Due to averaging, $U_{\text {ent }}$ cannot distinguish between C-tests with only a few highly uncertain gaps and Ctests which have a higher number of less uncertain gaps. We investigated another aggregation function $U_{\text {soft }}$ in preliminary experiments, which measures the entropy across all gaps and thus, is more robust to C-tests with only a few highly uncertain gaps.

Formulation. For our second formulation of $U$, we use a different aggregation method. Due to the mean, $U_{\text {ent }}$ is unable to distinguish between C-tests where the system is highly uncertain for only a few gaps and C-tests where all gaps are less, but more equally uncertain. We propose to use the softmax function $\sigma$ for normalizing $H\left(g_{i}\right)$ and then to compute the entropy across all gaps $g_{i}$. $U_{\text {soft }}$ thus considers the distribution of gap-uncertainties and favours C-tests with equally distributed gapuncertainties over C-tests with only a few highly uncertain gaps.

$$
U_{\text {soft }}(x)=\gamma\left[-\sum_{i=1}^{n} \sigma_{i}\left(H\left(g_{i}\right)\right) \log \sigma_{i}\left(H\left(g_{i}\right)\right)\right]
$$

As the squashing of the individual gap entropy values removes the information about their magnitude, we furthermore scale the resulting value by the normalized mean entropy

$$
\gamma=\frac{1}{n \log n} \sum_{i=1}^{n} \frac{H\left(g_{i}\right)}{H_{\max }}
$$

for all gaps $g_{i}$ in the C-test.

Results. Figure 11 shows similar tendencies as we already found for $U_{\text {ent }}$ in section 6 . Again, we can observe that random sampling performs better in early iterations, while the other sampling strategies outperform it in latter iterations. Averaging the accuracy across all iterations (table 5) shows that both our joint sampling strategies tos and comb again perform in average better than the other sampling strategies for the static, motivated, and interrupted learners. However, conducting a Wilcoxon signed-rank test with $p<0.05$ shows that the active learning curves only significantly differ for the static learner.

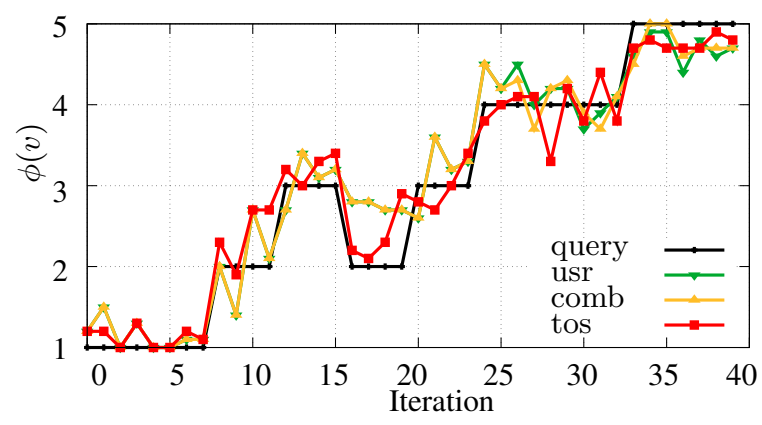

Figure 8: Sampled instances for the interrupted learner using $U_{\text {soft }}$.

For the user objective (also shown in table 5) we observe that all strategies which include a user objective significantly outperform rand and unc, but there is no clear favorite amongst them. This can also be seen in figure 8 where all strategies manage to sample instances close to the queried difficulty (in black). 

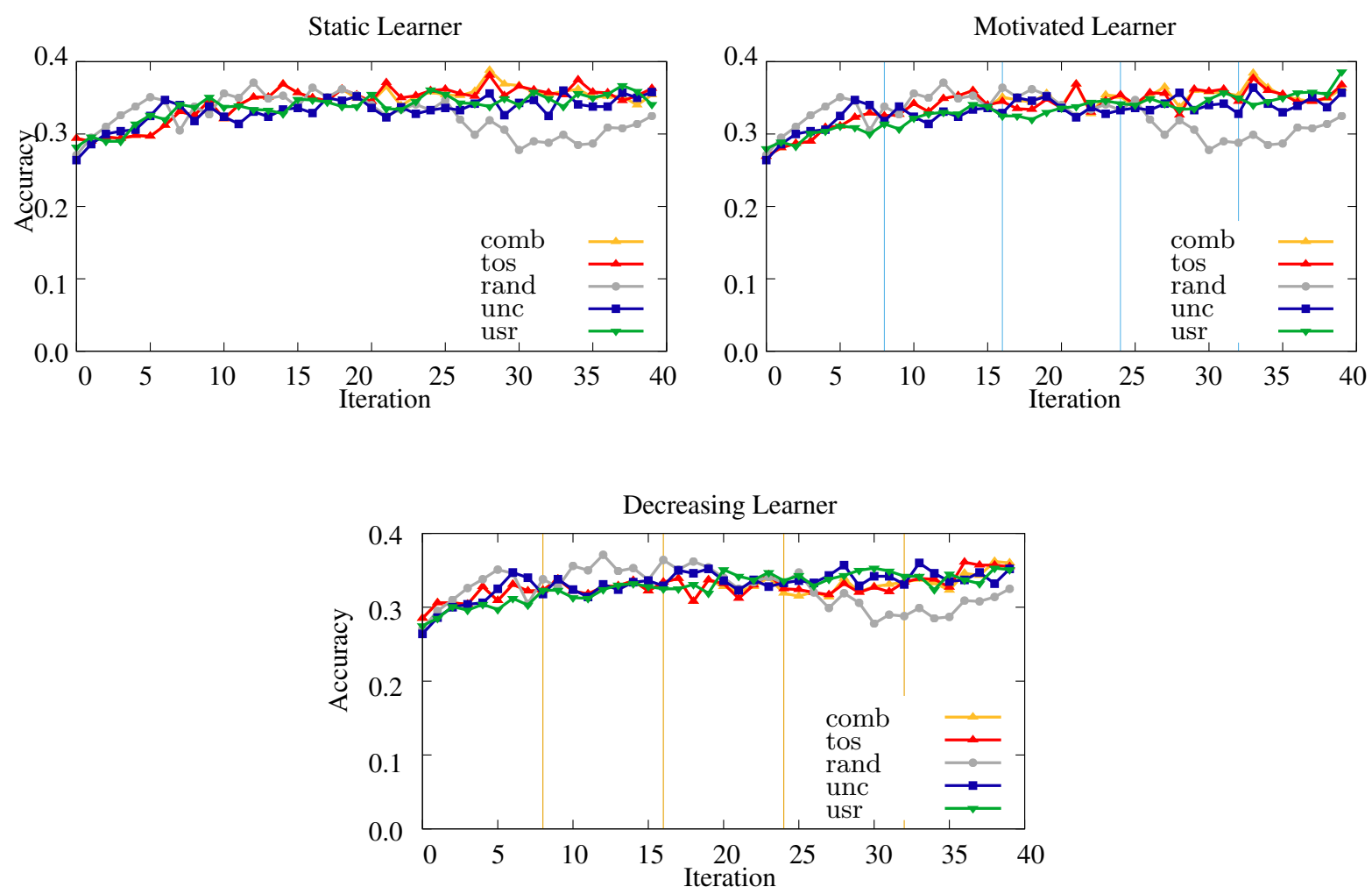

Figure 6: Accuracy scores for the static, motivated, and artificially decreasing learners using $U_{\text {ent }}$.
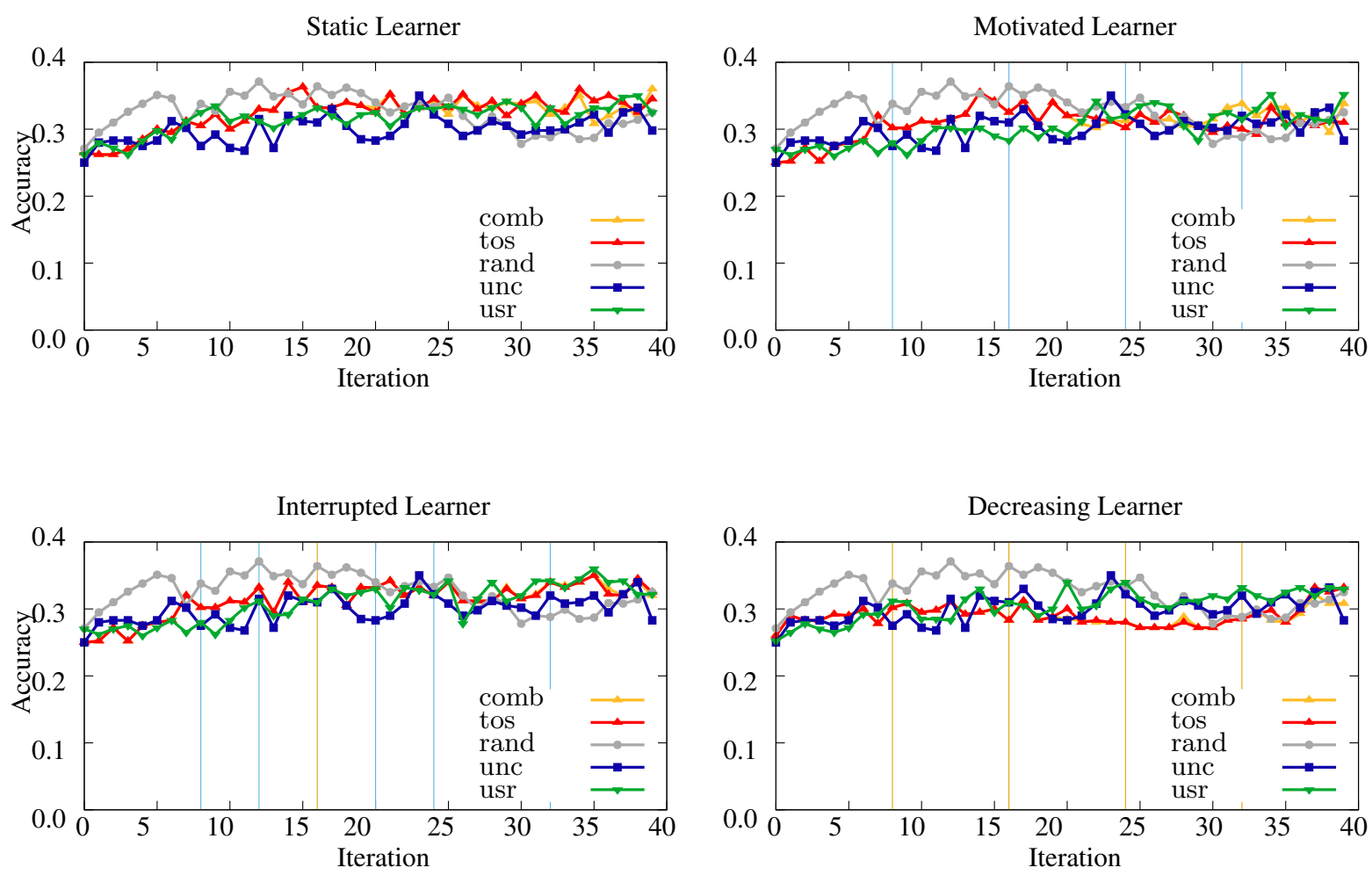

Figure 7: Accuracy on the test data for $U_{\text {soft }}$. 


\section{A.3 Impact of the aggregation function}

Figure 9 compares both our aggregation functions $U_{\text {ent }}$ and $U_{\text {soft }}$ against each other on the interrupted learner for uncertainty, combined, and trade-off sampling. Although $U_{\text {ent }}$ and $U_{\text {soft }}$ differ to some regard, directly comparing both aggregation functions and the respective aggregated scores (cf., table 5 shows that there is no clear favourite between both. Extensive work with respect to both aggregation functions as well as additional aggregation strategies remains to be investigated in future work.
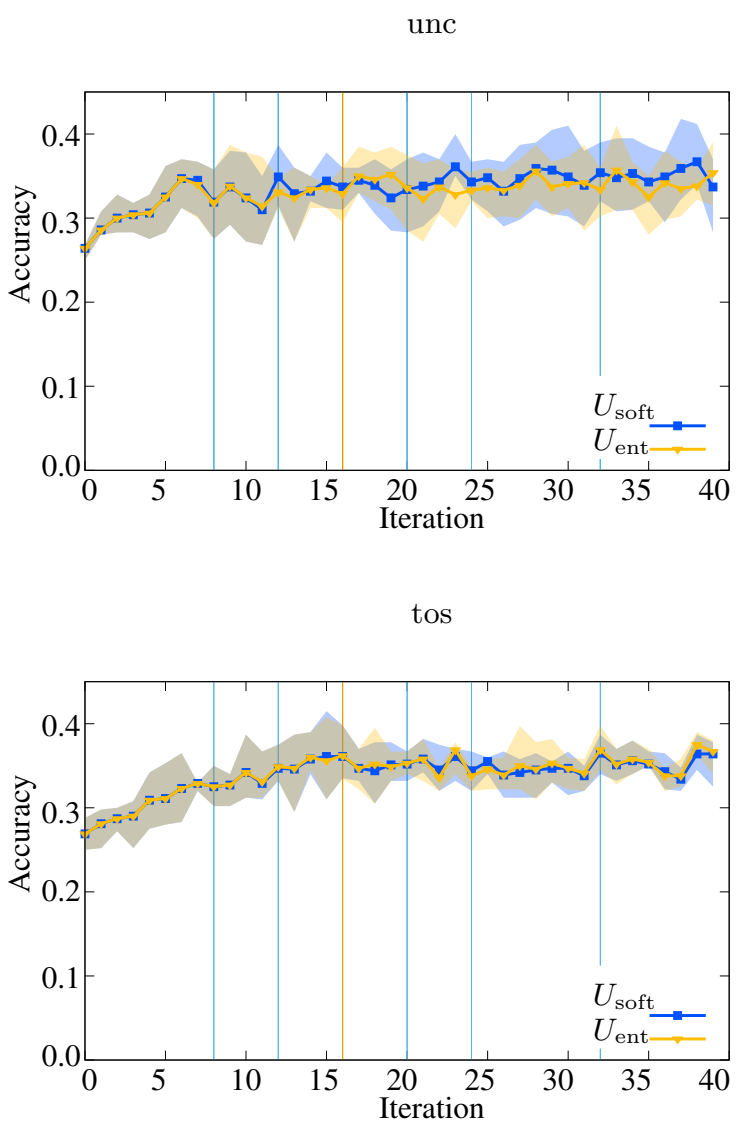

comb

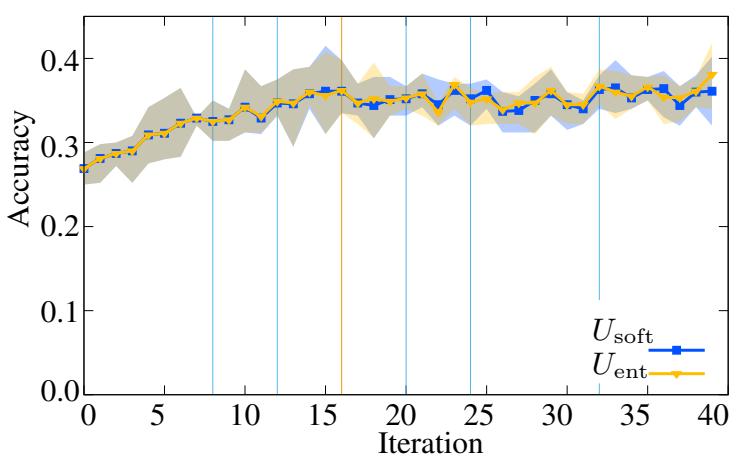

Figure 9: Comparing $U_{\text {ent }}$ and $U_{\text {soft }}$ for the interrupted learner.

\section{A.4 Stability of system objective}

To provide estimates how stable our approaches are across different randomly initialized weights, we compute the upper and lower quartiles for each sampling strategy across all runs. Figures 10 and 11 show our results for the interrupted learner.

Overall, we observe that user-oriented sampling has lower deviations across different runs for both our aggregation functions $U_{\text {ent }}$ and $U_{\text {soft }}$. One reason for this may be that in contrast to uncertainty sampling, we query instances with highly certain predictions in our user-oriented sampling approach. This leads to sampled instances which are easier to learn resulting in a higher training stability with small data. Comparing the user-oriented against our joint sampling strategies shows that especially in the earlier iterations, our proposed sampling strategies perform better and provide more stable training.

\section{A.5 Further investigation of $\lambda$}

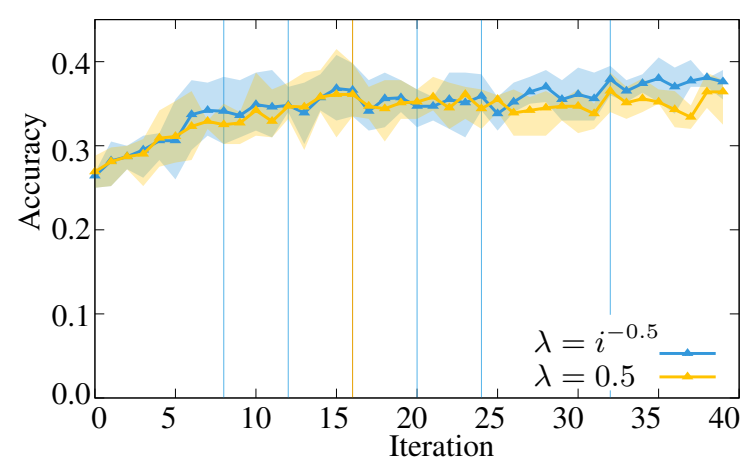

Figure 12: Accuracy of trade-off sampling for annealed and fixed $\lambda$ using $U_{\text {soft }}$ for the interrupted learner.

To further validate our findings for an annealed $\lambda$, we conduct the same experiments with our novel aggregation function $U_{\text {soft }}$. As with $U_{\text {ent }}$, we obtain significant improvements for our trade-off sampling strategy (figure 12) for the motivated and interrupted learner, but also a significant decrease for the static and decreasing learner. With respect to the user objective, we do not see any significant differences at all, indicating that $U_{\text {soft }}$ does not benefit at all from the emphasised user objective in later iterations.

Table 5 (including the previous results for better comparability) shows the results for all learner behaviours and both our aggregation functions $U_{\text {ent }}$ 

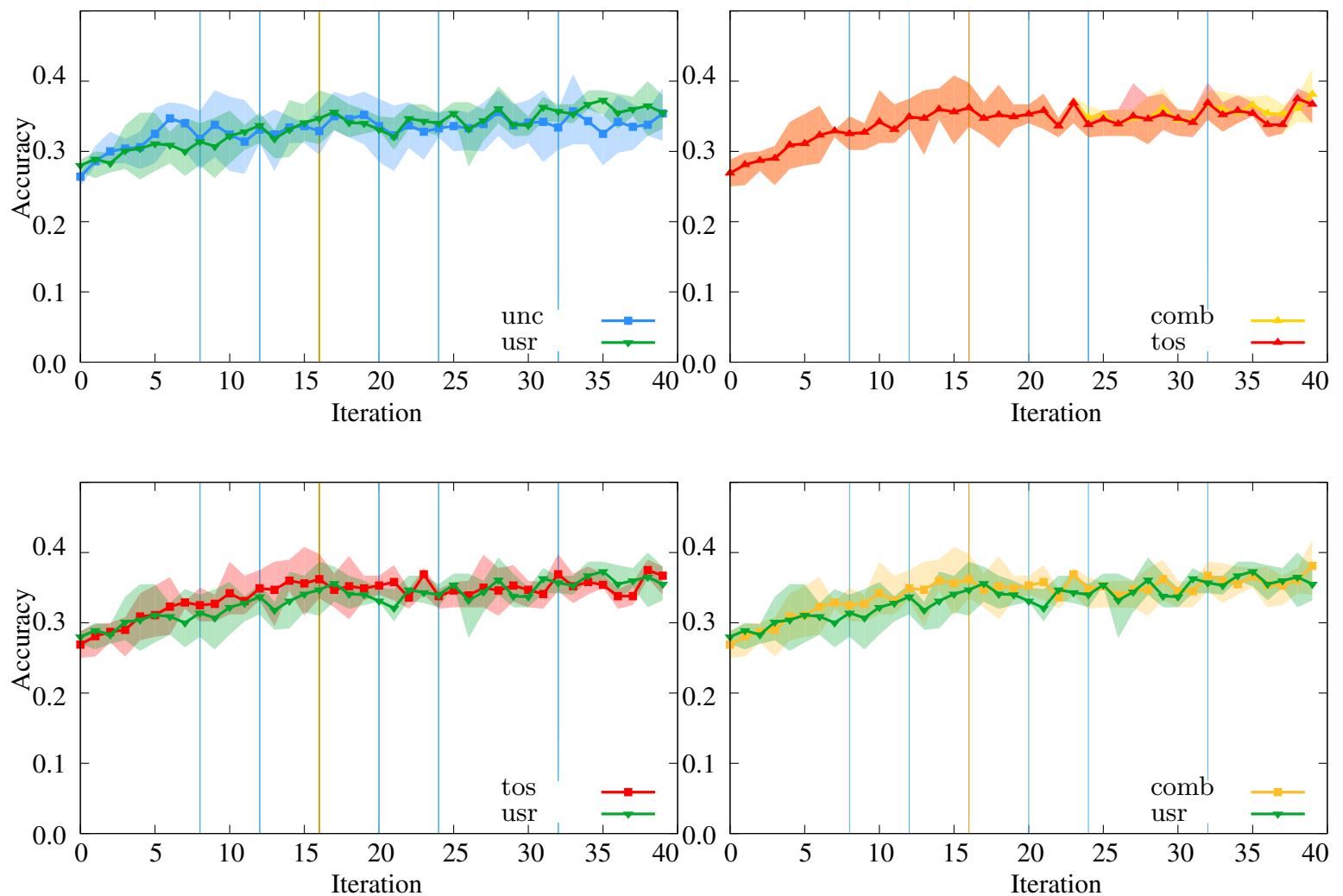

Figure 10: Upper and lower quartiles for the interrupted learner using $U_{\text {ent }}$.

\begin{tabular}{|c|c|c|c|c|c|c|c|c|c|c|c|c|c|c|c|c|}
\hline & \multicolumn{8}{|c|}{$\mathrm{U}_{\text {ent }}$} & \multicolumn{8}{|c|}{$\mathrm{U}_{\text {soft }}$} \\
\hline & \multicolumn{4}{|c|}{ Accuracy } & \multicolumn{4}{|c|}{ MAE } & \multicolumn{4}{|c|}{ Accuracy } & \multicolumn{4}{|c|}{ MAE } \\
\hline & STAT & MOT & INT & DEC & STAT & MOT & INT & DEC & STAT & MOT & INT & DEC & STAT & MOT & $\Gamma$ INT & DEC \\
\hline $\operatorname{tos}_{\lambda}$ & .333 & .346 & .347 & .314 & 0.85 & 0.53 & 0.48 & 0.53 & .331 & .345 & .347 & .316 & 0.86 & 0.64 & 0.59 & 0.70 \\
\hline tos & .334 & .338 & .339 & .327 & 0.98 & 0.65 & 0.93 & 0.75 & .345 & .336 & .338 & .327 & 0.91 & 0.64 & 0.62 & 0.70 \\
\hline comb & .343 & .340 & .341 & .327 & 0.98 & 0.63 & 0.88 & 0.65 & .344 & .338 & .340 & .326 & 0.93 & 0.63 & 0.62 & 0.66 \\
\hline usr & .338 & .331 & .334 & .328 & 0.85 & 0.58 & 0.65 & 0.75 & .337 & .331 & .334 & .328 & 0.92 & 0.63 & 0.59 & 0.70 \\
\hline unc & .332 & .331 & .331 & .331 & 1.17 & 1.33 & 1.35 & 1.72 & .336 & .336 & .336 & .335 & 1.24 & 1.32 & 1.31 & 1.72 \\
\hline rand & .325 & .325 & .325 & .325 & 1.16 & 1.22 & 1.82 & 1.24 & .325 & .325 & .325 & .325 & 1.16 & 1.22 & 1.82 & 1.24 \\
\hline
\end{tabular}

Table 5: Averaged accuracy and MAE for all strategies (including the annealed $\lambda$ strategy) for $U_{\text {ent }}$ and $U_{\text {soft }}$.

and $U_{\text {soft. }}$. As can be seen, using an annealed $\lambda$ $\left(\operatorname{tos}_{\lambda}\right)$ leads to the best results with respect to the user objective for $U_{\text {ent }}$ but fails to improve the results for $U_{\text {soft. }}$. 

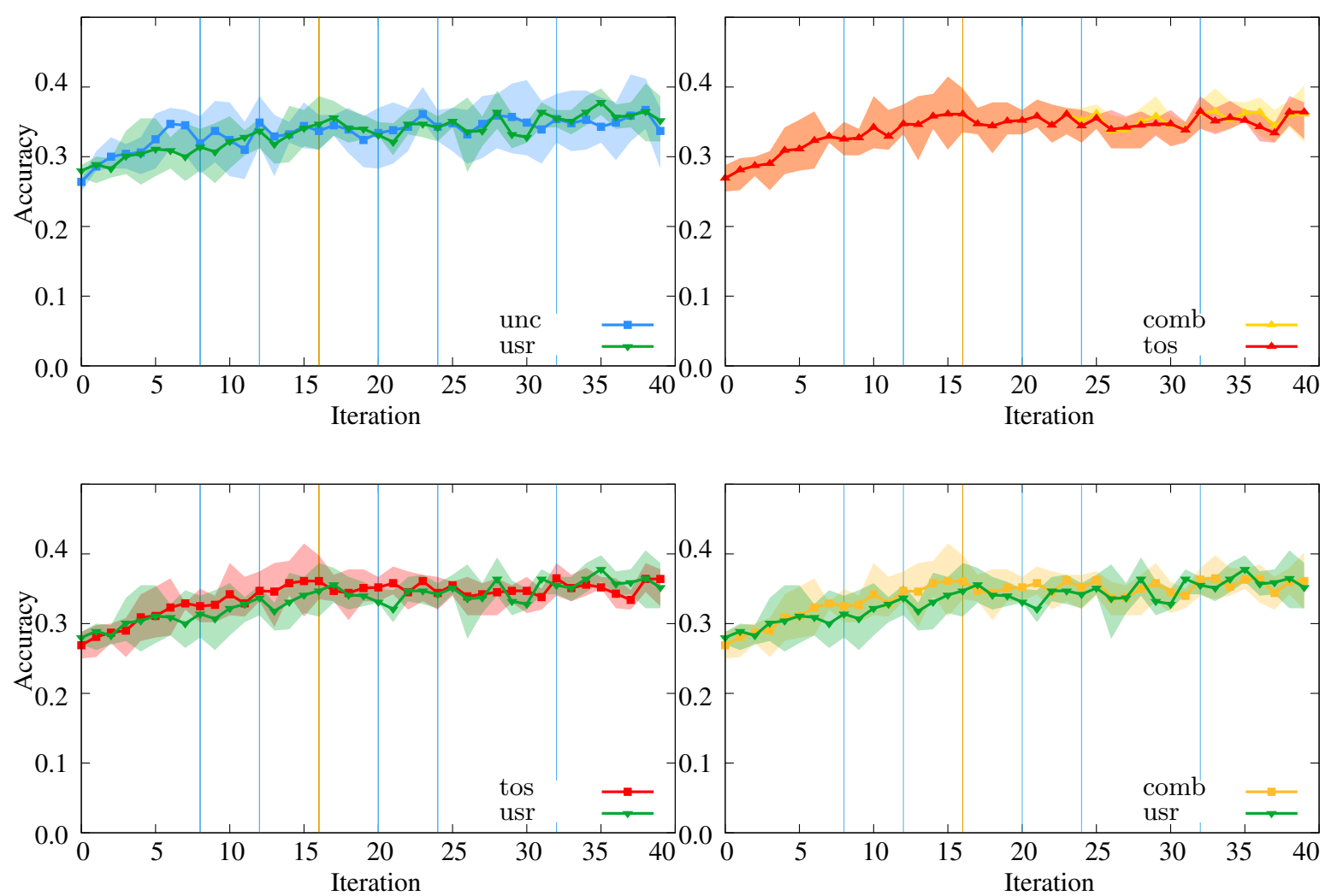

Figure 11: Upper and lower quartiles for the interrupted learner using $U_{\text {soft }}$. 